\title{
Mitochondrial Alterations with Mitochondrial DNA Depletion in the Nerves of AIDS Patients with Peripheral Neuropathy Induced by 2'3'-Dideoxycytidine (ddC)
}

\author{
Marinos C. Dalakas, Cristina Semino-Mora, and Marta Leon-Monzon \\ Neuromuscular Diseases Section, National Institute of Neurological Disorders and Stroke, National Institutes of \\ Health, Bethesda, Maryland
}

\begin{abstract}
SUMMARY: The 2'3'-dideoxycytidine (ddC), a nonazylated dideoxynucleoside analog used for the treatment of AIDS, causes a dose-dependent, painful, sensorimotor axonal peripheral neuropathy in up to $30 \%$ of the patients. To investigate the cause of the neuropathy, we performed morphological and molecular studies on nerve biopsy specimens from well-selected patients with ddC-neuropathy and from control subjects with disease, including patients with AIDS-related neuropathy never treated with ddC. Because ddC, in vitro, inhibits the replication of mitochondrial DNA (mtDNA), we counted the number of normal and abnormal mitochondria in a $0.04 \mathrm{~mm}^{2}$ cross-sectional area of the nerves and quantified the copy numbers of mtDNA by competitive PCR in all specimens. A varying degree of axonal degeneration was present in all nerves. Abnormal mitochondria with enlarged size, excessive vacuolization, electron-dense concentric inclusions and degenerative myelin structures were prominent in the ddC-neuropathy and accounted for $55 \% \pm 2.5 \%$ of all counted mitochondria in the axon and Schwann cells, compared with $9 \%$ $\pm 0.7 \%$ of the controls $(p<0.001)$. Significantly $(p<0.005)$ reduced copy numbers, with as high as $80 \%$ depletion, of the mtDNA was demonstrated in the nerves of the ddC-treated patients compared with the controls. We conclude that ddC induces a mitochondrial neuropathy with depletion of the nerve's mtDNA. The findings are consistent with the ability of ddC to selectively inhibit the $\gamma$-DNA polymerase in neuronal cell lines. Toxicity to mitochondria of the peripheral nerve is a new cause of acquired neuropathy induced by exogenous toxins and may be the cause of neuropathy associated with the other neurotoxic antiretroviral drugs or toxic-metabolic conditions. (Lab Invest 2001, 81:1537-1544).
\end{abstract}

\begin{abstract}
$Z$ alcitabine (ddC) is a synthetic $2^{\prime} 3^{\prime}$-dideoxynucleoside analog commonly used for the treatment of patients infected with acquired immunodeficiency syndrome (AIDS) (Fischl et al, 1993; Mitsuya and Broder, 1986; Yarchoan et al, 1988). Since the time it was first introduced for the treatment of AIDS, ddC was causally connected with a dose-dependent, painful, sensorimotor axonal peripheral neuropathy (Berger et al, 1993; Blum et al, 1996; Dubinsky et al, 1989; Lewis and Dalakas, 1995; Moyle and Sadler, 1998). The neuropathy occurs in $34 \%$ of patients receiving $\mathrm{ddC}$, and it is a limiting factor in the use of this drug in the treatment of AIDS (Dalakas, 2001; Dubinsky et al, 1989; Berger et al, 1993; Lewis and Dalakas, 1995; Blum et al, 1996; Moyle and Sadler, 1998). It is manifested approximately 6 to 8 weeks after the initiation of therapy and usually improves 3 to 4 weeks after discontinuation of the drug (Berger et al, 1993; Blum et al, 1996; Dalakas, 2001; Dubinsky et al, 1989; Lewis and Dalakas, 1995; Moyle and Sadler, 1998). In conjunction with other neurotoxic risk factors
\end{abstract}

Received August 29, 2001.

Address reprint requests to: Dr. Marinos C. Dalakas, Chief, Neuromuscular Diseases Section, NINDS, NIH, Building 10, Room 4N248, Bethesda, MD 20892. or antiretroviral agents, the incidence of ddCassociated neurotoxicity appears increased (Benbrik et al, 1997; Dalakas, 2001; Moyle and Sadler, 1998).

The mechanism by which ddC causes neuropathy or exacerbates subclinical neuropathy in patients with HIV infection is unclear. Although electrophysiological studies have been consistent with an axonal neuropathy affecting sensory and motor axons (Berger et al, 1993; Blum et al, 1996; Dalakas, 2001; Dubinsky et al, 1989; Lewis and Dalakas, 1995; Moyle and Sadler, 1998), the targeted organelle in the axon or the Schwann cell responsible for the dysfunction of the nerves has not been examined. Because ddC belongs to the family of nucleoside analogs that exert, in vitro, a unique toxicity to mitochondria by terminating the mitochondrial DNA chain (Benbrik et al, 1997; Chen and Cheng, 1989; Chen et al, 1991; Dalakas, 2001; Keilbaugh et al, 1993; Lewis and Dalakas, 1995; Luster et al, 1989), we examined in this study nerve biopsy specimens from carefully selected AIDS patients with ddC-related neuropathy for alterations in the morphology of the mitochondria and the amount of the nerve's mitochondrial DNA (mtDNA). Nerve biopsies from AIDS patients with HIV-related neuropathy never treated with ddC and from patients with other axonal neuropathies served as controls. 


\section{Results}

\section{Light Microscopy}

With toluidine-blue, a varying degree of axonal dropout and axonal degeneration was noted in all the diseased nerves, regardless of cause. No unique feature characteristic of each neuropathy (ddC, HIV, other) was noted. The degree of axonal degeneration and formation of myelin ovoids, however, appeared prominent in the ddC-nerves (Fig. 1).

\section{Electron Microscopy}

Abnormalities in the Mitochondria of Myelinated and Unmyelinated Axons. Vesicular degeneration, dense lamellar bodies, myelin debris, and several elongated processes separating the myelin sheaths were prominent in the ddC-treated nerves (not shown). Ovoids were observed as a mixture of disintegrated myelin segments and degenerated axonal components, consistent with Wallerian degeneration. The neurofilaments appeared normal. A rather striking finding was the presence of vacuolizations in the axonal mitochondria of both the myelinated (Fig. 2, A to D) and unmyelinated (Fig. 3A) fibers, with severe interruption of the inner mitochondrial morphology, enlarged size, and, at times, electron-dense osmiophilic deposits between the outer and inner limiting membranes and the cristae (Fig. 2, A to D).

Because abnormal mitochondria can occur as a reaction to the disease process and may not be specific for ddC-treated nerves, a quantification was performed in all studied specimens including the disease controls. The total number of mitochondria in the axoplasm was not different in the three groups and averaged $82.7 \pm 2.5$ per nerve. The percentage of abnormal mitochondria in the axoplasm of the nerves from the ddC-treated patients was $54.86 \% \pm 2.47 \%$, compared with $27.77 \% \pm 4.97 \%(p<0.05)$ in the axoplasm of the nerves from the HIV-neuropathy patients and $9.04 \% \pm 0.66 \%$ in the other neuropathy controls $(p<0.001)$ (Table 1$)$.

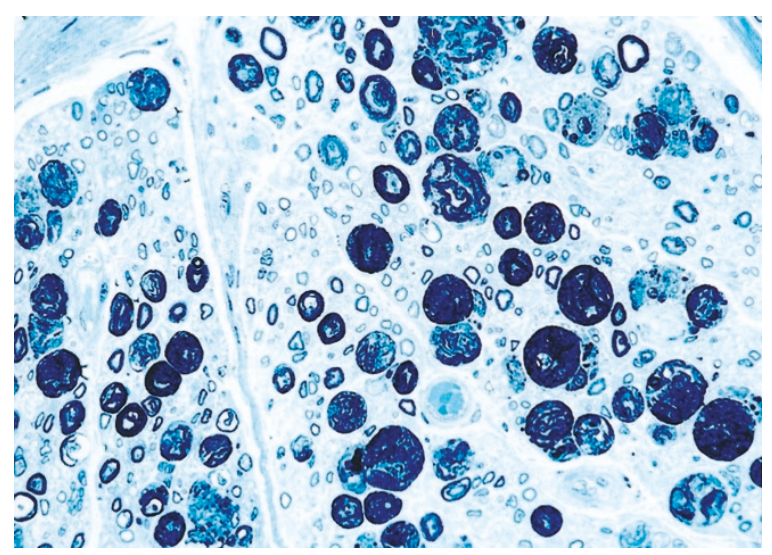

Figure 1.

Cross thick sections of a nerve biopsy from a ddC-neuropathy patient stained with toluidine blue demonstrates axonal drop-out and several ovoid structures caused by ongoing axonal degeneration. $(\times 200)$
Abnormalities in the Schwann cells. The presence of abnormal mitochondria was also the hallmark alteration in the Schwann cells (Fig. 3B). Electron-dense deposits were noted between the membranes and in the cristae in a pattern similar to the one described in the axoplasm. Elongated or round dense structures resembling paracrystalline inclusions were also seen inside the vacuolated mitochondria. Mitochondria with only one outer membrane, suggesting a degenerative process, were occasionally noted (Fig. 3, A and B). The total number of counted mitochondria in the Schwann cells averaged $53 \pm 2.4$ per nerve in all specimens. The percentage of abnormal mitochondria in the Schwann cells accounted for $47.11 \% \pm 5.36 \%$ of all the mitochondria in the ddC-treated patients, compared with $14.30 \% \pm 1.82 \%$ in the disease control nerves $(p<0.05)$ and $19.44 \% \pm 5.24 \%$ in the HIV-neuropathy nerves $(p<0.05)$ (Table 1).

\section{Mitochondrial DNA}

Competitive PCR demonstrated a significant reduction in the amount of mtDNA in the nerves of the ddC-treated patients compared with the neuropathy controls. In the $2 \mu \mathrm{g}$ of total nerve DNA, the average number of mtDNAs found in the neuropathy controls was $1 \times 10^{9} \pm 0.04$ copies, compared with $5.2 \times 10^{7}$ \pm 0.16 DNA copies $(p<0.005)$ in the nerves of the ddC-treated patients. In Figure $4 A$, representative competitive PCR electrophoretic products for three nerve biopsies from ddC-treated patients (a to $c$ ) demonstrate mtDNA depletion compared with two neuropathy controls ( $d$ and e). Competition equivalent points for each of the corresponding specimens (Fig. $4 \mathrm{~B}, \mathrm{a}^{\prime}$ to $\left.\mathrm{e}^{\prime}\right)$, determined on plots of the calculated OD ratio of the amplified signals (OD for the competitive DNA:OD for the mtDNA-derived product) versus the logarithm of the competitive DNA copy numbers added sequentially to each reaction, demonstrate a linear correlation. Similar results were obtained with the PCR products obtained with the duplicate samples at high and low PCR cycles (data not shown).

\section{Discussion}

Sural nerve biopsies from patients with ddCassociated neuropathy demonstrate a significantly higher incidence of abnormal mitochondria in their axon and Schwann cells with depletion of mtDNA, compared with diseased controls. The findings complement the effect of this drug in inhibiting the replication of mtDNA in neuronal cell lines in vitro and indicate that ddC, as used in AIDS patients, causes a mtDNA-depleting neuropathy.

A small number of abnormal mitochondria, ranging from $1 \%$ to $10 \%$, can be seen in the axon or the Schwann cells of the sural nerves from patients with various neuropathies (Schroder and Sommer, 1991). Whether these changes are primary or secondary and whether they affect cell function is unknown. Even in patients with mitochondrial encephalomyelopathies, where peripheral neuropathy has been observed and 

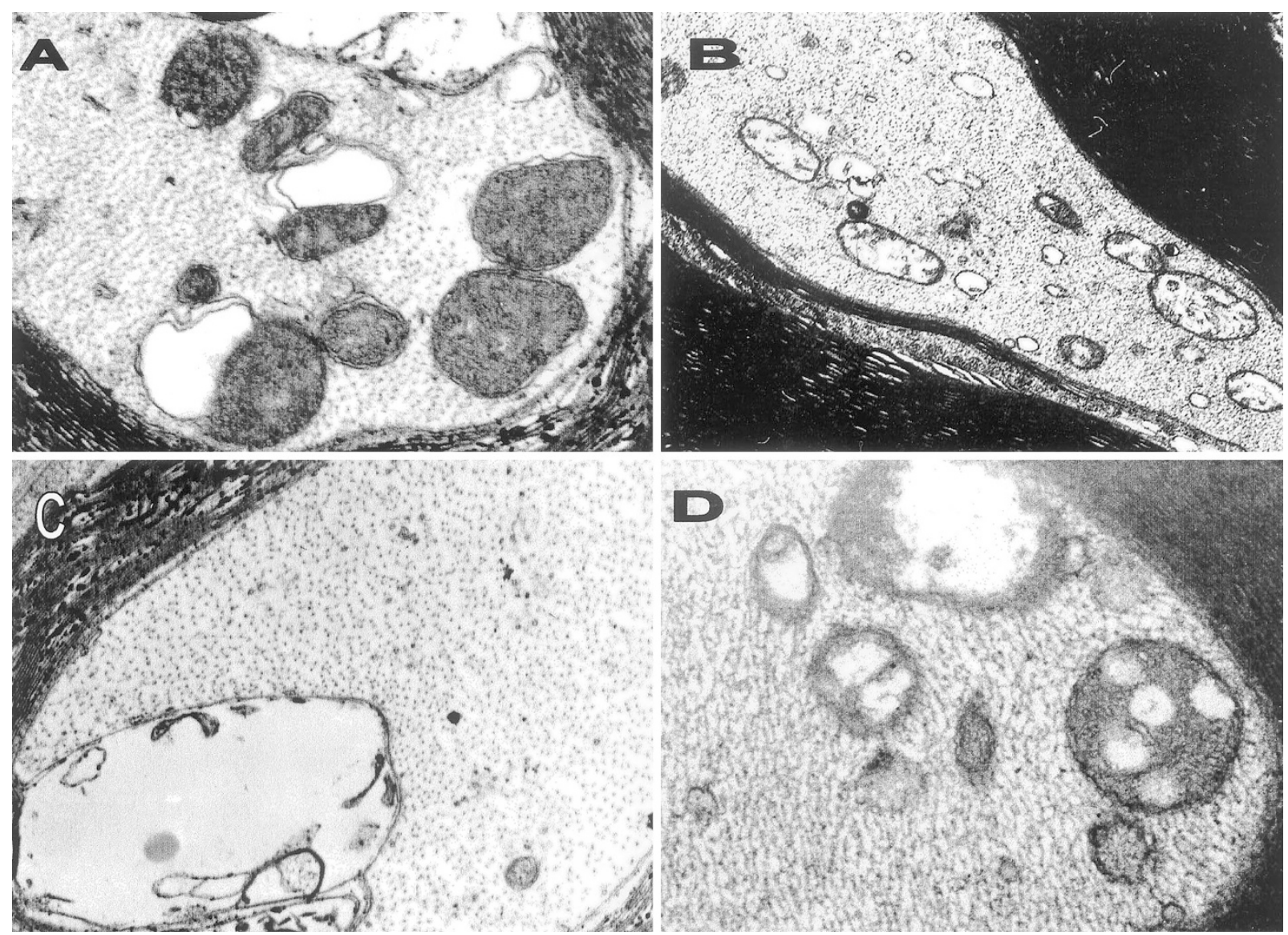

\section{Figure 2.}

Electron microscopy of sural nerve biopsies from patients with ddC-neuropathy demonstrate abnormal mitochondria in the myelinated axons (A to D). Vacuolization, with loss of cristae and disorganized matrix is prominent in several mitochondria. Some of the mitochondria are swollen with small and large vacuoles (D) and others contain dense inclusions $(C)$ or are replaced by dense amorphous material (A and D). Magnification: $A, \times 37,500 ; B, \times 16,500 ; C, \times 32,500 ; D, \times 37,500$.

mutations in the muscle mtDNA have been noted (Eymard et al, 1991; Johns, 1995; Pezeshkpour et al, 1987; Yiannikas et al, 1986), morphological or molecular studies have been largely focused on the muscle, not the nerve. The presence of morphologically abnormal mitochondria in number, shape, size, and cristae in the sural nerve biopsies of patients with neuropathies, especially in HIV-infected individuals, may be related to the following: (a) non-disease-specific degradation, secondary to axonal degeneration; (b) compensatory effect as an adaptation response of the mitochondria to increase their size and enzymatic respiratory activity; (c) various neurotoxic factors including the neurotoxic potential of HIV itself; and (d) an artifactual effect of the fixation process. In our cases, these possibilities have been excluded by quantifying the mitochondria in the ddC-treated patients and disease controls, processing all the nerves in our laboratory using the same methodology, selecting as disease controls nerve biopsies from patients with other, even more severe, axonal neuropathies, and, most importantly, including nerve biopsy specimens from HIV-positive patients never treated with ddC and confirming the abnormal morphology with molecular studies.
The observed morphological and molecular abnormalities in the peripheral nerve mitochondria of the ddC-treated patients complement observations obtained in experimental animals and neuronal cell lines treated with ddC. Healthy rabbits fed ddC exhibit abnormal mitochondria in their Schwann cells (mitochondrial Schwannopathy) and dorsal root ganglia, intramyelinic edema, axonal degeneration, and impaired Schwann cell metabolism with reduced level of myelin Po mRNA (Anderson et al, 1992; Feldman and Anderson, 1994; Feldman et al, 1992). In cell lines, ddC, like its two sister nucleoside analogs, AZT and ddl, inhibits the $\gamma$-DNA polymerase of the mitochondrial matrix and terminates, noncompetitively, the nascent mtDNA causing impairment of DNA replication and termination of the mtDNA chain. This, in turn, leads to mtDNA depletion with dysfunction of mtDNAencoded enzymes (Arnaudo et al, 1991; Benbrik et al, 1997; Chen and Cheng, 1989; Chen et al, 1991; Cupler et al, 1995; Dalakas et al, 1990, 1994; Keilbaugh et al, 1993; Lewis and Dalakas, 1995; Luster et al, 1989; Mhiri et al, 1991). The demonstrated effect of ddC in causing a "mitochondrial DNA-depleting neuropathy" is analogous to the known effect of AZT in causing a "mitochondrial DNA-depleting myopathy" (Arnaudo et 

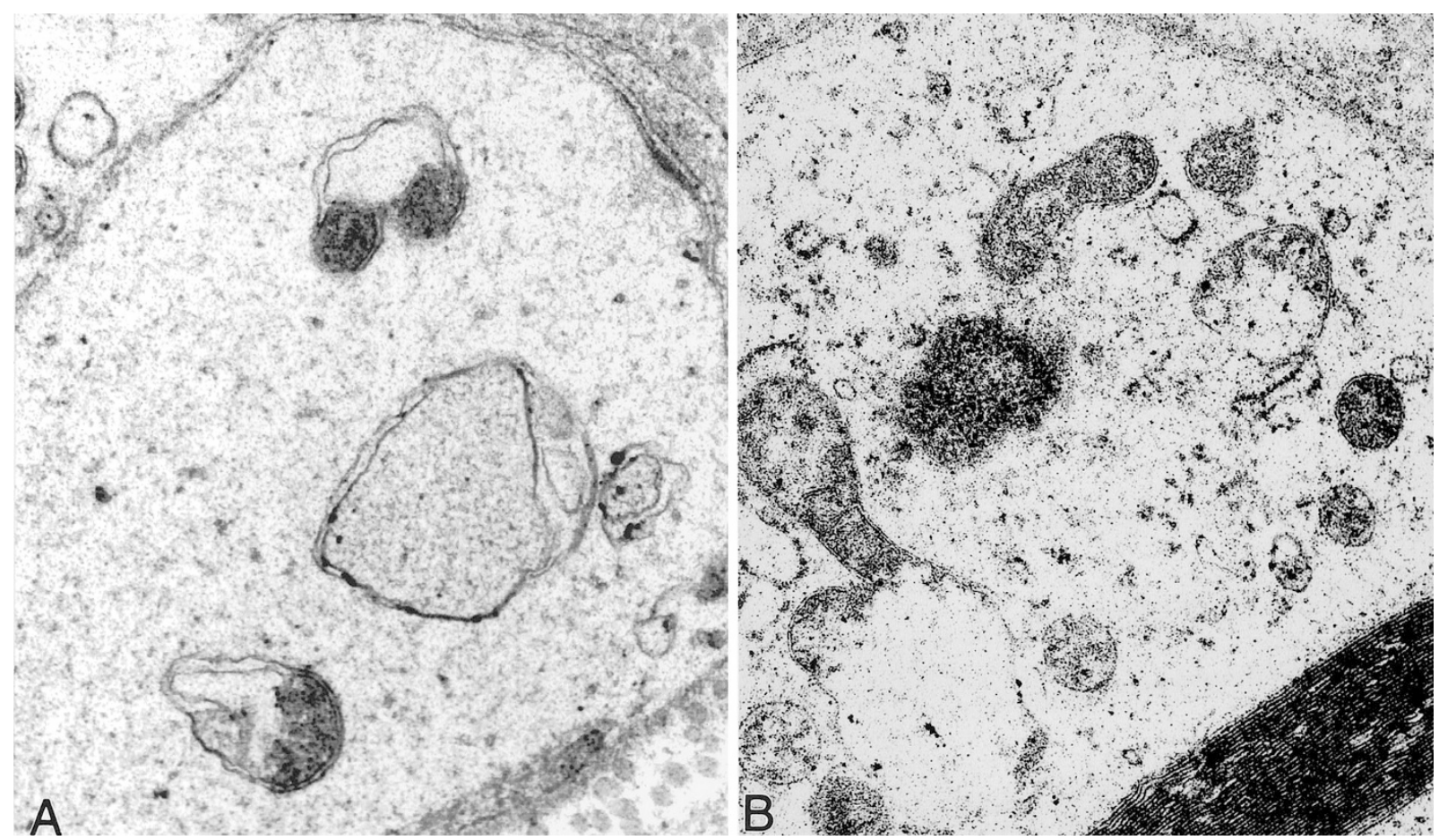

Figure 3.

Electron microscopy of sural nerve biopsies from a patient with ddC-neuropathy demonstrates abnormal mitochondria in an unmyelinated axon (A) and Schwann cells (B). Vacuolization with loss of cristae or dense inclusions (A) are prominent. Magnification: $A, \times 23,000 ; B, \times 29,100$.

Table 1. Percentage of Abnormal Mitochondria Observed in the Axons and Schwann Cells in the Sural Nerve Biopsies of Patients Treated with ddC Compared with Those of Diseased Control Subjects

\begin{tabular}{lcc}
\hline & \multicolumn{2}{c}{ Percentage of abnormal mitochondria ${ }^{a}$} \\
\cline { 2 - 3 } Patients with neuropathy & In the axoplasm & In the Schwann cells \\
\hline ddC-treated & $54.86 \pm 2.47^{b}$ & $47.11 \pm 5.36^{b}$ \\
HIV $(+)$ control & $27.77 \pm 4.97$ & $19.44 \pm 5.24$ \\
Other, HIV $(-)$ controls & $9.04 \pm 0.66$ & $14.30 \pm 1.82$ \\
\hline
\end{tabular}

\footnotetext{
${ }^{a}$ Mitochondria, counted in the axons or the Schwann cells in $15.93 \pm 1.52$ myelinated and unmyelinated axons per nerve, were compared between the ddC-treated patients and the other HIV $(-)$ or HIV $(+)$ neuropathy controls.

${ }^{b}$ The number of abnormal mitochondria was significantly higher $(p<0.001)$ in the ddC-induced neuropathy compared with the other two neuropathy controls.
}

al, 1991; Bozzette et al, 1991; Cupler et al, 1995; Dalakas et al, 1990, 1994; Jay et al, 1994; Lamperth et al, 1991; Lewis et al, 1992, 1994; Mhiri et al, 1991). The preferential organ toxicity of these nucleosides appears to be related to the thymidine kinase isoforms responsible for their phosphorylation, or to tissuespecific polymorphisms of mtDNA polymerase- $\gamma$ (Lewis and Dalakas, 1995). In vitro, ddC and ddl cause inhibition of mtDNA in the neuronal PC12 cell line, but not in the Friend erythroleukemia cell line. In contrast, AZT is toxic to the Friend cell line but not the PC12 cell line (Chen and Cheng, 1989; Chen et al, 1991; Keilbaugh et al, 1993; Luster et al, 1989). In humans, AZT is myotoxic (Arnaudo et al, 1991; Cupler et al, 1995; Dalakas et al, 1990, 1994; Lamperth et al, 1991; Lewis and Dalakas, 1995; Lewis et al, 1992, 1994; Mhiri et al, 1991) but not neurotoxic (Bozzette et al, 1991), whereas ddC and ddl are neurotoxic but not myotoxic (Jay et al, 1994). In healthy rats and rabbits, ddC causes an axonal neuropathy with abnormal mito- chondria in the Schwann cells, but no signs of myopathy (Anderson et al, 1992; Bozzette et al, 1991; Cupler et al, 1995; Dalakas et al, 1990, 1994; Feldman and Anderson, 1994; Feldman et al, 1992; Jay et al, 1994; Lamperth et al, 1991; Lewis et al, 1994; Mhiri et al, 1991; Russel et al, 1995). In contrast, rats treated with AZT develop a mitochondrial myopathy, but not neuropathy (Lamperth et al, 1991; Lewis et al, 1992, 1994).

The mitochondrial abnormalities observed in both the myelinated and unmyelinated fibers in the sural nerve biopsies of patients with ddC-neuropathy can explain the painful, predominantly sensory symptoms experienced by these patients. The partial reversibility of the neuropathy, when the drug is discontinued (Dubinsky et al, 1989), is supported by the molecular events of mtDNA inhibition and the phenomenon of heteroplasmy. As discussed for the AZT-associated myopathy (Arnaudo et al, 1991; Dalakas et al, 1990; Lewis and Dalakas, 1995), ddC is incorporated only in 

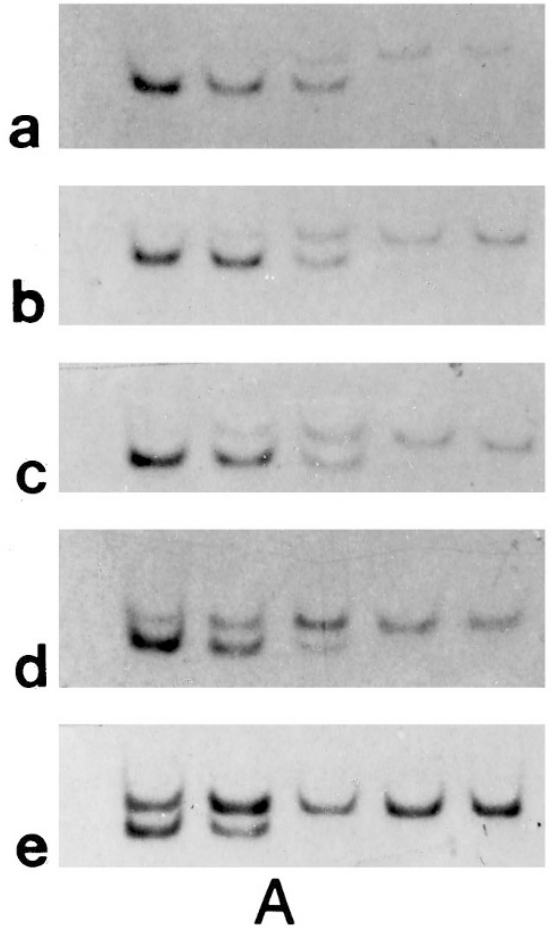
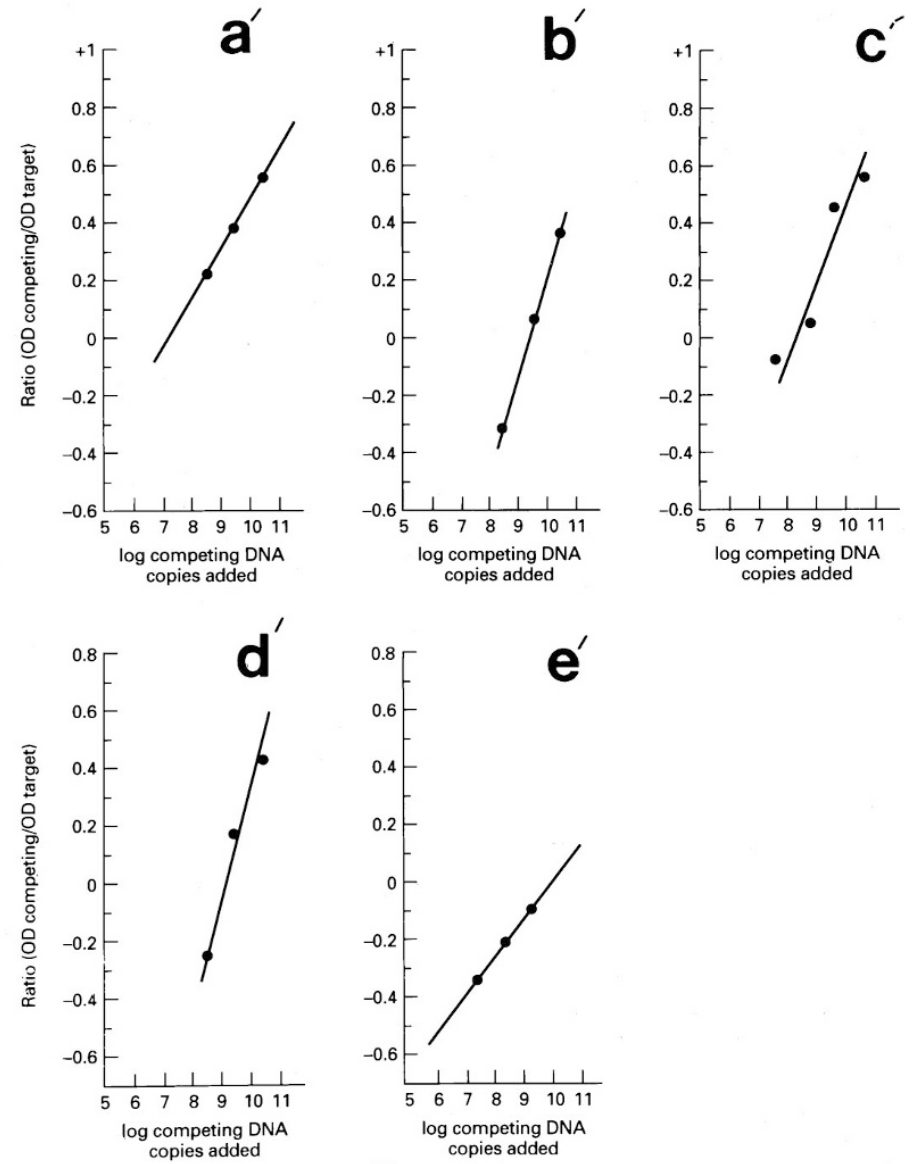

$\mathrm{B}$

Figure 4.

$A$ and B, Reactions of competitive PCR analysis ( $A, a$ to e) and their corresponding plots of quantified copy numbers of mtDNA (B, a' to e') for each specimen. A, A constant amount of total nerve DNA was competitively amplified from the nerves of HIV/ddC patients (a to $\mathrm{c}$ ) and diseased control nerves ( $d$ and $\mathrm{e}$ ). The top band corresponds to mtDNA of $182 \mathrm{bp}$ in length amplified from each specimen; the bottom band corresponds to the decreasing amount (from left to right) of the $156 \mathrm{bp}$ competitive DNA. In the presence of a low concentration of mtDNA, as in the three nerve biopsies from the ddC-treated patients, the template competitive DNA is preferentially amplified (a to c). In contrast, in the presence of a normal amount of mtDNA, as in the control nerves, the intensity of the amplified mtDNA is similar to the competitive DNA template $(c$ to d). B, The plots represent the competition equivalence points of the calculated optical density (OD) ratio of the amplified PCR signal (for the competitive DNA template:mtDNA) versus the logarithm of the number of the competitive DNA copies added to each reaction. Each plot in $B$ (a' to $\mathrm{e}^{\prime}$ ) corresponds to the PCR amplified product from the same patient shown in A (a to e). A linear correlation was obtained between the OD ratios and the sequentially decreasing number of added competitive DNA copies.

the DNAs of the replicating mitochondria resulting at a given time in normal and abnormal mtDNA and allowing for partial recovery upon discontinuation of the drug. The effect of ddC on replicating mitochondria most likely affects oxidative phosphorylation, as supported by studies on mitochondrial function in vitro (Keilbaugh et al, 1991) and in vivo (Chariot and Gherardi, 1991), thereby resulting in a shortage of energy within the Schwann cells or axons and nerve dysfunction.

The present observations are of practical significance for several reasons. First, they provide a mechanism by which ddC causes neuropathy and opens the avenue for exploring therapeutic approaches by removing phosphorylated ddC from the mtDNA terminus, possibly by the exonucleases associated with DNA pol- $\gamma$ (Lewis and Dalakas, 1995), or by agents that compete with the mitochondrial enzymatic systems, such as uridine and pyruvate (Keilbaugh et al,
1993). Second, they unravel a new category of mitochondrial neuropathies that need to be investigated as a possible cause of certain hereditary or acquired toxic and metabolic neuropathies. Thallium, a heavy metal that causes toxic neuropathy in man, has been shown to cause selective enlargement and disruption of the mitochondria in the axons of the peripheral nerve fibers (Spencer et al, 1973). In diabetic neuropathy, there is emerging evidence of toxicity to mitochondria (E. Feldman, personal communication, 2001). Finally, the study supports the implication that the signs of systemic toxicity manifested with neuropathy, lipodystrophy, lactic acidosis, steatosis, and pancreatorenal syndrome, observed with the currently used highly active antiretroviral therapy (HAART) therapy, may be related to a general effect on mitochondrial function (Brinkman et al, 1999; Carr and Cooper, 2000; Miller et al, 2000) that resembles the hereditary mitochondrial neurological disorders in which neurop- 
athy is also common (Dalakas, 2001; Lewis and Dalakas, 1995; Moraes and DiMauro, 1990).

\section{Methods}

\section{Patients}

ddC-Associated Neuropathy. Four patients, ages 38 to 52 (mean, 45 years) were HIV-positive for a mean period of 56.7 months (range, 20 to 76 months). Their mean CD4+ cell count was 242 (range, 61 to 422). They developed neuropathy manifested by painful sensory symptomatology in the feet that began approximately 6 to 10 weeks after starting ddC therapy. By the time a sural nerve biopsy was performed, they had received a mean cumulative ddC dose of $545 \mathrm{gm}$ (range, 236 to $1012 \mathrm{gm}$ ) for a mean period of 8.2 months (range, 3 to 15 months). The neuropathy was confirmed by electrophysiological studies that demonstrated absent, or very low amplitude, sural nerve potentials, reduced amplitude of the peroneal or posterior tibial nerves, and denervation potentials with needle electromyography. Because this was not a prospective study, conduction studies were not available before ddC treatment to determine whether a subclinical AIDS-related neuropathy was aggravated by ddC. However, before initiation of ddC therapy, all the studied patients denied any neuropathic symptoms, and no abnormal neurological signs were recorded by the primary care physician. In addition, in all four patients, the neuropathic symptoms began 6 to 10 weeks after ddC therapy, a pattern typically seen with ddC-related neuropathy (Berger et al, 1993; Blum et al, 1996; Dalakas, 2001; Dubinsky et al, 1989; Fischl et al, 1993; Lewis and Dalakas, 1995; Moyle and Sadler, 1998; Yarchoan et al, 1988). Other causes of neuropathy, such as diabetes, alcohol, drug abuse, nutritional factors, low B12 levels, or exposures to other neurotoxins, were excluded. The patients were studied before 1996, and none of them had received protease inhibitors. All the patients signed an informed consent and had a sural nerve biopsy processed as described later.

Neuropathy Control Patients. Sural nerve biopsies from three patients with AIDS-associated neuropathy never treated with ddC were concurrently studied. These patients developed peripheral neuropathy, which was axonal in two and mixed, axonal with secondary demyelinating features, in the other. Two of the patients were not receiving any treatment at the time of the biopsy, but the third had been previously treated with $A Z T$, which has been associated with toxic myopathy, but not with neuropathy (Benbrik et al, 1997; Dalakas, 2001; Dalakas et al, 1998; Lewis and Dalakas, 1995). In addition, sural nerve biopsies from three patients with non-HIV-related axonal polyneuropathy of varying severity were processed as described later.

\section{Nerve Biopsy Preparations}

One piece of the sural nerve was fresh-frozen and processed for molecular studies. The other piece was embedded in Spurr and processed for electron microscopy and quantification of the mitochondria. The following methodology was used.

Light and Electron Microscopy. A piece of nerve was fixed immediately by immersion in $2.5 \%$ glutaraldehyde in $0.1 \mathrm{M}$ cacodylate buffer for 4 hours at room temperature, postfixed in $1 \%$ osmium tetroxide for 1.5 hours, dehydrated in increasing concentrations of alcohol, immersed in propylene oxide, and embedded in Spurr epoxy resin at $60^{\circ} \mathrm{C}$. Thick sections (1.5 to 3 $\mu \mathrm{m})$ were stained with toluidine blue and thin sections $(500 \Delta)$ were stained with uranyl acetate and lead citrate. The thick sections were examined under a Zeiss light microscope and the grids with the thin sections were examined in a JEOL 100CX electron microscope (Akishima, Japan). Transversely fascicular fragments were embedded in different blocks. Three blocks were examined from each peripheral nerve specimen.

Quantification of Mitochondria. Ultrathin, crosssections, from each block were placed in three copper square grids (200 mesh), each containing 20 sections with transverse fascicles. Mitochondria, identified on the basis of double membrane, were counted in the Schwann cells and the axons of myelinated and unmyelinated fibers on the screen of the electron microscope at $\times 13,000$, as previously described (SeminoMora et al, 1994). Randomly selected axons (mean, $15.93 \pm 1.52$ ) were counted in a $0.04 \mathrm{~mm}^{2}$ area in all specimens. Abnormal mitochondria were defined on the basis of (a) vacuolization or giant size with paucity of cristae, (b) presence of electron-dense or concentric inclusions with membrane-like myelin formations, (c) elongated or round-dense structures resembling paracrystalline inclusions, and (d) osmiophilic deposits between the inner and outer membranes or the cristae. The number of abnormal mitochondria was expressed as the percentage among all the counted mitochondria in the aforementioned areas (SeminoMora et al, 1994). The number of abnormal mitochondria per axon or Schwann cell within the counted areas was also calculated.

Quantification of Mitochondrial DNA by Competitive $P C R$. The competitive PCR was used to quantify copy numbers of mtDNA. This method was selected because it allows the incorporation of decreasing amounts of competitive hybrid DNA template to compete with a fixed amount of DNA samples containing an unknown number of mtDNA copies. The competitive hybrid DNA template was synthesized using total DNA extracted from the peripheral blood mononuclear cells of an HIV-positive patient following the original method of Zhang et al (1994), which was used to detect mtDNA depletion in the lymphocytes caused by nucleoside analogs. For the competitive DNA template synthesis, two 40-mer hybrid primers were constructed containing specific mtDNA sequences. One primer, Mimic 06, contained the mt06 20-mer sequence derived from the D-loop region, with an additional 20-mer sequence identical to the nucleotide 5953-5952 fragment of the tat/rev region of the HIVcDNA strand; the other primer, Mimic 187, contained 
the mt187 20-mer length sequence of the D-loop region, with an additional 20-mer sequence complementary to nucleotide 6049-6068 fragment of the tat/rev of the HIV-cDNA strand. The competitive DNA template was synthesized by mixing in a PCR reaction HIV-peripheral blood lymphocyte (PBL)-DNA with primers Mimic 06 and Mimic 187, as described (Zhang et al, 1994). This reaction amplified a 156 bp product that was analyzed by agarose gel electrophoresis and purified by column chromatography. The number of copies of the competitive DNA template was determined by the optical density (OD) at $260 \mathrm{~nm}$; the MW of the product was $95.7 \mathrm{kd}$. The competitive DNA template consisted of HIV tat/rev sequences flanked by mtDNA sequences at each end of the template. In the presence of mt-, the mt06 and mt187 primers produce an amplification product of $182 \mathrm{bp}$ in length with a MW of $111 \mathrm{kd}$, which allows the distinction between mtDNA and template DNA.

For the quantification of mt-DNA, total DNA was extracted from the human nerve biopsy specimens of the ddC-treated patients and the other controls. The DNA was dissolved in sterile water and the concentration of each preparation was determined by the $O D$ at $260 \mathrm{~nm}$. DNA/PCR was performed following standard conditions using (a) a fixed $2 \mu \mathrm{g}$ of total nerve cellular DNA, (b) a decreasing amount of competitive DNA template $(30,3,0.3,0.03$, and $0 \mathrm{ng})$ corresponding to $3.1 \times 10^{10}, 3.1 \times 10^{9}, 3.1 \times 10^{8}$, and $3.1 \times 10^{7}$ DNA copies, (c) mitochondria primers (mt06 and $\mathrm{mt108}$ ), and (d) 10 microcurie of ${ }^{35} \mathrm{~S}$-ATP in the reaction mixture, as described (Zhang et al, 1994). Reactions omitting total nerve DNA or competitive DNA template were also run as controls (data not shown). Five reactions in five separate tubes were set for each specimen. The mitochondria primers 06 and 187 amplified two bands, 156 and 182 bp each, which were visualized by ethidium bromide staining. Gels were then dried, exposed to x-ray film, and scanned using a Bio-Rad scanner and an imaging integrator program (Bio-Rad Laboratories, Hercules, California) to determine for each reaction the concentration of the DNA in each band (Zhang et al, 1994). To validate the results, two sets of amplification reactions with 20 and 35 cycles were performed. To determine the copy number of mtDNA present in the amplification product, the OD ratio of the band obtained with the competing DNA (156 bp) to the OD of the band of the target DNA (182 bp) was plotted against the logarithm of the number of copies of the competing DNA added to each reaction, as described (Zhang et al, 1994). When the densities of both bands are identical, the ratio is 1 and the logarithm is 0 . The logarithm of the number of copies present in the fixed specimens of 2 $\mu \mathrm{g}$ was calculated from the $\mathrm{X}$ axis when the $\mathrm{Y}$ axis equals 0 .

\section{References}

Anderson TD, Davidovich A, Arceo R, Brosnan C, Arezzo J, and Schaumburg $\mathrm{H}$ (1992). Peripheral neuropathy induced by 2'3'-dideoxycytidine neurotoxicity. Lab Invest 66:63-74.
Arnaudo E, Dalakas MC, Shanske S, Moraes CT, DiMauro S, and Echon EA (1991). Depletion of muscle mitochondrial DNA in AIDS patients with zidovudine-induced myopathy. Lancet 337:508-510.

Benbrik E, Chariot P, Bonavaud S, Ammi-Said M, Frisdal E, Rey C, Gherardi R, and Barlovatz-Meimon G (1997). Cellular and mitochondrial toxicity of zidovudine (AZT), didanosine (ddl) and zalcitabine (ddC) on cultured human muscle cells. J Neurol Sci 149:19-25.

Berger AR, Arezzo JC, Schaumburg HH, Skowron G, Merigan T, Bozzette S, Richman D, and Soo (1993). W 2'3'dideoxycytidine (ddC) toxic neuropathy: A study of 52 patients. Neurology 43:358-362.

Blum AS, Dal Pan GJ, Feinberg J, Raines C, Mayjo K, Cornblath DR, and McArthur JC (1996). Low-dose zalcitabine-related toxic neuropathy: Frequency, natural history and risk factors. Neurology 46:999-1003.

Bozzette SA, Santangelo J, Villasana D, Fraser A, Wright B, Jacobsen C, Hayden E, Schnack J, Spector SA, and Richman DD (1991). Peripheral nerve function in persons with asymptomatic or minimally symptomatic HIV disease: Absence of zidovudine neurotoxicity. J Acquir Immune Defic Syndr 4:851-855.

Brinkman K, Smeitink JA, Romijin JA, and Reiss P (1999). Mitochondrial toxicity induced by nucleoside-analogue reverse-transcriptase inhibitors is a key factor in the pathogenesis of antiretroviral-therapy-related lipodystrophy. Lancet 354:1112-1115.

Carr A and Cooper DA (2000). Adverse effects of antiretroviral therapy. Lancet 356:1423-1430.

Chariot P and Gherardi R (1991). Partial cytochrome c oxidase deficiency and cytoplasmic bodies in patients with zidovudine myopathy. Neuromuscul Disord 1:357-363.

Chen C, Vazquez-Padua M, and Cheng Y (1991). Effect of anti-human immunodeficiency virus nucleoside analogs on mitochondrial DNA and its implications for delayed toxicity. Mol Pharmacol 39:625-628.

Chen C-H and Cheng Y-C (1989). Delayed cytotoxicity and selective loss of mitochondrial DNA in cells treated with the anti-human immunodeficiency virus compound 2'3'dideoxythymidine. J Biol Chem 264:11934-11937.

Cupler EJ, Danon MJ, Jay C, Hench K, and Ropka MC (1995). Early zidovudine associated myopathy: Histopathological features and clinical correlations. Acta Neuropathol 90:1-6.

Dalakas MC (2001). Peripheral neuropathy and antiviral drugs. J Peripher Nerv Syst 6:14-20.

Dalakas MC, Illa I, Pezeshkpour GH, Laukaitis JP, Cohen B, and Griffin J (1990). Mitochondrial myopathy caused by long-term zidovudine therapy. N Engl J Med 332:1098-1105.

Dalakas MC, Leon-Monzon ME, Bernardini I, Gahl WA, and Jay CA (1994). The AZT-induced mitochondrial myopathy is associated with muscle carnitine deficiency and lipidstorage. Ann Neurol 35:482-487.

Dalakas MC, Yarchoan R, Spitzer R, and Sever JL (1998). Treatment of HIV-related polyneuropathy with 3'-azido-2',3'dideoxythimidine (AZT). Ann Neurol 23:92-94.

Dubinsky RM, Yarchoan R, Dalakas M, and Broder S (1989). Reversible axonal neuropathy from the treatment of AIDS and related disorders with $2{ }^{\prime} 3^{\prime}$-dideoxycytidine (ddC). Muscle Nerve 12:856-860. 
Eymard B, Penicaud A, Leger JM, Romero N, Marsac C, Fardeau M, and Brunet $P$ (1991). Peripheral nerve in mitochondrial disease: clinical and electrophysiological data. A study of 28 cases. Rev Neurol 147:508-512.

Feldman D and Anderson TD (1994). Schwann cell mitochondrial alterations in peripheral nerves of rabbits treated with 2',3'-dideoxycytidine. Acta Neuropathol 87:71-80

Feldman D, Brosnan C, and Anderson T (1992). Ultrastructure of peripheral neuropathy induced in rabbits by $2^{\prime}, 3^{\prime}-$ dideoxycytidine. Lab Invest 66:75-85.

Fischl MA, Olson RM, Follansbee SE, Lalezari JP, Henry DH, Frame PT, Remick SC, Salgo MP, Lin AH, and Nauss-Karol C (1993). Zalcitabine compared with zidovudine in patients with advanced HIV-1 infection who received previous ziduvidine therapy. Ann Intern Med 118:762-769.

Jay C, Ropka M, and Dalakas MC (1994). The drugs 2'3'dideoxyinosine (ddl) and $2{ }^{\prime} 3$ '-dideoxycytidine (ddC) are safe alternatives in people with AIDS with zidovudine-induced myopathy. J Acquir Immune Defic Syndr 7:630-631.

Johns DR (1995). Mitochondrial DNA and disease. N Eng J Med 333:638-644.

Keilbaugh SA, Hobbs GA, and Simpson MV (1993). Antihuman immunodeficiency virus type 1 therapy and peripheral neuropathy: Prevention of 2'3'-dideoxycytidine toxicity in PC12 cells, a neuronal model, by uridine and pyruvate. Mol Pharmacol 44:702-706.

Keilbaugh SA, Prusoff WH, and Simpson MV (1991). The PC12 cell as a model for studies of the mechanism of induction of peripheral neuropathy by anti-HIV-1 dideoxynucleoside analogs. Biochem Pharmacol 42:R5-R8.

Lamperth L, Dalakas MC, Dagani F, Anderson J, and Ferri R (1991). Abnormal skeletal and cardiac muscle mitochondrial induced by zidovudine (AZT) in human muscle in vitro and in an animal model. Lab Invest 65:742-751.

Lewis W and Dalakas MC (1995). Mitochondrial toxicity of antiviral drugs. Nat Med 1:417-422.

Lewis W, Gonzalez B, Chomyn A, and Papoian T (1992). Zidovudine induces molecular, biochemical, and ultrastructural changes in rat skeletal muscle mitochondria. J Clin Invest 89:1354-1360.

Lewis W, Simpson JF, and Meyer RR (1994). Cardiac mitochondrial DNA polymerase- $\gamma$ is inhibited competitively and noncompetitively by phosphorylated zidovudine. Circ Res 74:344-348.

Luster MI, Germolec DR, White KL, Fuchs BA, Fort MM, Tomaszewski JE, Thompson M, Blair PC, McCay JA, and Munson AE (1989). A comparison of three nucleoside analogs with anti-retroviral activity on immune and hematopoietic functions in mice: In vitro toxicity to precursor cells and microsomal environment. Toxicol Appl Pharmacol 101:328339.

Mhiri C, Baudrimont M, Bonne G, Geny C, Degoul F, Marsac C, Roullet E, and Gherardi R (1991). Zidovudine myopathy: a distinctive disorder associated with mitochondrial dysfunction. Ann Neurol 29:606-614.
Miller KD, Cameron M, Wood LV, Dalakas MC, and Kovacs JA (2000). Lactic acidosis and hepatic steatosis associated with use of stavudine: Report of four cases. Ann Intern Med 133:192-196.

Mitsuya $\mathrm{H}$ and Broder S (1986). Inhibition of the in vitro infectivity and cytophatic effect of human T-lymphotrophic virus type III/lymphadenopathy-associated virus (HTLV-III/ LAV) by 2'3'-dideoxynucleosides. Proc Nat Acad Sci USA 83:1911-1915.

Moraes CT and DiMauro S (1990). Quantitative defects of mitochondrial DNA. In: DiMauro $S$ and Wallace DC, editors. Mitochondrial DNA in human pathology. New York: Raven Press, 97-108.

Moyle GJ and Sadler M (1998). Peripheral neuropathy with nucleoside antiretrovirals: Risk factors, incidence and management. Drug Saf 19:481-94.

Pezeshkpour G, Krarup C, Buchthal F, DiMauro S, Bresolin $\mathrm{N}$, and McBurney $\mathrm{J}$ (1987). Peripheral neuropathy in mitochondrial disease. J Neurol Sci 77:285-304.

Russel JW, Cupler EJ, and Dalakas MC (1995). Electrophysiological and pathological changes in 2'3'-Dideoxycytidine (ddC)-induced neuropathy in an animal model (abstract). Ann Neurol 38:306.

Schroder JM and Sommer C (1991). Mitochondrial abnormalities in human sural nerves: Fine structural evaluation of cases with mitochondrial myopathy, hereditary and nonhereditary neuropathies, and review of the literature. Acta Neuropathol 82:471-482.

Semino-Mora MC, Leon-Monzon M, and Dalakas MC (1994). Effect of L-carnitine on the zidovudine-induced destruction of human myotubes. Part I: L-carnitine prevents the myotoxicity of AZT in vitro. Lab Invest 71:102-112.

Spencer PS, Peterson ER, Madrid AR, and Raine CS (1973). Effects of thallium salts on neuronal mitochondria in organotypic cord-ganglia-muscle combination cultures. J Cell Biol 58:79-95.

Yarchoan R, Perno CF, Thomas RV, Klecker RW, Allain JP, Wills RJ, McAtee N, Fischl MA, Dubinsky R, and McNeely MC (1988). Phase I studies of $2^{\prime} 3^{\prime}$-dideoxycitidine in severe human immunodeficiency virus infection as a single agent and alternating with zidovudine (AZT). Lancet 1:76-81.

Yiannikas C, McLeod JG, Pollard JD, and Baverstock J (1986). Peripheral neuropathy associated with mitochondrial myopathy. Ann Neurol 20:249-257.

Zhang H, Cooney DA, Sreenath A, Zhan Q, Agbaria R, Stowe EE, Fornace AJ Jr, and Johns DG (1994). Quantitation of mitochondrial DNA in human lymphoblasts by a competitive polymerase chain reaction method: Application to the study of inhibitors of mitochondrial DNA content. Mol Pharmacol 46:1063-1069. 